\title{
Gold nanotriangles decorated with superparamagnetic iron oxide nanoparticles: a compositional and microstructural study
}

\author{
J. A. Hachtel, S. Yu, A.R. Lupini, S. T. Pantelides, M. Gich, A. Laromaine, A. Roig
}

The combination of iron oxide and gold in a single nanoparticle results in both magnetic and plasmonic properties that can stimulate novel applications in bio-sensing, medical imaging, or therapeutics. Microwave assisted heating allows the fabrication of multi-component, multi-functional nanostructures by promoting selective heating at desired sites. Recently, we reported a microwave-assisted polyol route yielding gold nanotriangles decorated with iron oxide nanoparticles. Here, we present an in-depth microstructural and compositional characterization of the system by using scanning transmission electron microscopy (STEM) and electron energy loss (EELS) spectroscopy. A method to remove the iron oxide nanoparticles from the gold nanocrystals and some insights on crystal nucleation and growth mechanisms are also provided.

\section{Introduction}

Colloidal synthesis of multi-material nanocrystals with selectable morphologies, beyond core-shell and dimer geometries, is progressing fast in nanocrystal research ${ }^{1,2,3}$. Novel uses of multi-material anisotropic nanocrystals are expected in fields ranging from nanomedicine to photocatalysis ${ }^{4,5,6}$. Specifically, the combination of gold and iron oxide in a single nanoparticle (NP) exhibiting both magnetic and plasmonic properties has been proposed for applications related to bio-sensing, medical imaging or therapeutics ${ }^{7-10}$. Superparamagnetic iron oxide nanoparticles (SPIONs) are biocompatible and extensively validated for uses in nanomedicine, such as, magnetic resonance imaging ${ }^{11}$, drug delivery ${ }^{12}$, cell labeling ${ }^{13}$, bio-separation ${ }^{14}$ and hyperthermia ${ }^{15}$. On the other hand, the optical properties and the localized surface plasmon resonance (LSPR) of gold nanoparticles (Au NPs) have also motivated their biomedical uses in imaging ${ }^{16}$, sensing ${ }^{17}$, drug and gene delivery ${ }^{18}$ as well as in photothermal therapies ${ }^{19}$.

The shape $e^{20}$ and $\operatorname{size}^{21}$ of the Au NPs are crucial in determining the LSPR wavelength. Au NPs with anisotropic shapes displaying an absorption band in the near-infrared region (NIR) are appealing for photothermal therapies because NIR wavelengths can penetrate the biological tissues more efficiently ${ }^{22}$. Moreover, gold nanotriangles (Au NT) are highly effective when used in surface-enhanced Raman 
spectroscopy for biosensing ${ }^{23}$ and photothermal therapy ${ }^{24,25}$. Thus, fabrication methods for creating multi-component structures consisting of Au NPs with anisotropic planar shape (e.g. triangles and hexagons) and a magnetic component is gathering increased attention.

Most synthesis methods for gold nanoparticles with anisotropic shapes are based on a multi-step seedmediated approach using a cationic surfactant, most often the cetyltrimethylammonium bromide $(C T A B)^{23,26,27}$. The polyol process with ethylene glycol (EG) is another synthesis route used to generate seeds with different defects, such as multiple twin defects or stacking faults, yielding noble metal nanoparticles in the shape of dodecahedra or icosahedra (in the case of twins) and thin plates (in the case of stacking faults). The polyol route was initially employed for the fabrication of silver nanoparticles of different shapes and later the process was expanded to other systems, such as gold, platinum, rhodium and palladium nanoparticles ${ }^{28-30}$.

Recently, we reported a microwave-assisted polyol route yielding monodisperse Au NTs, decorated with a thin layer of SPIONs. These multi-material nanotriangles are readily dispersible in water, can selfassemble forming a monolayer at the liquid-air interface, display excellent magnetic response, and exhibit a LSPR band in the NIR region ${ }^{31}$. Here, we present an in-depth, nanoscale structural and compositional characterization of the system using scanning transmission electron microscopy (STEM) with electron energy loss spectroscopy (EELS) and provide insights on the early stages of crystal nucleation and growth. We present a technique to remove the SPIONs from the Au NPs post-synthesis, determine the phase of iron oxide present in the SPIONs, and find some suggestions that SPIONs might preferentially bond to different gold surfaces.

\section{Experimental}

\section{Materials}

Iron (III) acetylacetonate (Fe(acac)3 $\geq 97.0 \%$ ), polyvinylpyrrolidone (PVP, average molecular weight: $10000 \mathrm{~g} / \mathrm{mol}$ ), hydrogen tetrachloroaurate trihydrate ( $\mathrm{HAuCl} 4 \cdot 3 \mathrm{H} 2 \mathrm{O} \geq 99.9 \%$ ) were purchased from Sigma-Aldrich. Anhydrous benzyl alcohol (99\%) was from Scharlau and Ethylene glycol (EG $\geq 99 \%), \mathrm{HCl}$ (37\%) and acetone were bought from Panreac. All materials were used as-received without further purification. Milli-Q water (MQ-H2O) was used in all experiments. 


\section{Synthesis of PVP coated SPIONs}

SPIONs with a PVP surface coating (PVP-SPIONs) were synthesized following our microwave (MW) synthesis protocol ${ }^{32,33}$. Briefly, $680 \mathrm{mg}$ PVP were dissolved in $4 \mathrm{~mL}$ anhydrous benzyl alcohol by continuous sonication. Then, $12.36 \mathrm{mg} \mathrm{Fe}(\mathrm{acac}) 3(0.035 \mathrm{mmol})$ were mixed with the above prepared solution to give a homogenous solution of a dark red color. Solution was heated for $5 \mathrm{~min}$ at $60^{\circ} \mathrm{C}$ and $10 \mathrm{~min}$ at $180^{\circ} \mathrm{C}$ (reactor power at $250 \mathrm{~W}$ ). PVP-SPIONs (5 $\mathrm{nm}$ in diameter) were collected by adding 30 $\mathrm{mL}$ acetone, centrifuging at $1300 \mathrm{~g}$ for $30 \mathrm{~min}$ and redispersing in $4 \mathrm{~mL} E \mathrm{E}$ to be further used.

\section{Synthesis of hybrid Au-SPIONs nanoparticles}

$25 \mathrm{mg}$ of PVP were added to the as-obtained PVP-SPIONs dispersions in EG (4 mL) and sonicated to obtain a homogenous mixture where $40 \mu \mathrm{L}$ of $100 \mathrm{mM} \mathrm{HAuCl} 4$ were added. The molar ratio of free PVP to $\mathrm{HAuCl} 4$ is $0.625: 1$ and of SPIONs to $\mathrm{HAuCl} 4$ is $1.3: 1$. The solution was heated at $120 \mathrm{oC}$ for $10 \mathrm{~min}$ in the microwave reactor (reactor power at $250 \mathrm{~W}$ ). As-obtained Au-SPIONs were washed twice with acetone to remove free PVP and non-attached SPIONs, collected by centrifugation at 1300g for 2 min and redispersed in $2 \mathrm{~mL} \mathrm{MQ}-\mathrm{H} 2 \mathrm{O}$.

\section{Removal of SPIONs from Au NTs surface}

SPIONs were removed from the Au NPs surface using diluted $\mathrm{HCl}$ (1:10 dilution, $\mathrm{pH} \approx 3$ ). Au-SPIONs composites were concentrated by centrifugation at $3000 \mathrm{rpm}$ for $15 \mathrm{~min}$, after decanting the supernatant the concentrated composites were redispersed in $0.5 \mathrm{~mL}$ MiliQ water. $1 \mathrm{~mL}$ diluted $\mathrm{HCl}$ was then added dropwise to the system and incubated overnight. The acidic solution was replaced by fresh MiliQ water and the system was used for further characterization.

\section{Materials characterization}

Hydrodynamic sizes were determined with a Zetasizer Nano ZS (Malvern) with a He/Ne $633 \mathrm{~nm}$ laser at an angle $=173^{\circ}$ at $25^{\circ} \mathrm{C}$. Ultraviolet-visible-near infrared (UV-Vis-NIR) spectra were collected on a Cary5000 UV-Vis-NIR spectrophotometer. Superconductive Quantum Interference Device (SQUID) measurements were recorded on a magnetometer (Quantum Design MPMS5XL). The magnetization curves of the PVP-SPIONs and the hybrid Au NT-SPIONs were recorded at $5 \mathrm{~K}$ as a function of applied magnetic field. The morphologies of the AU-SPIONs were analysed in a JEOL JEM-1210 electron microscope operating at $120 \mathrm{KV}$. High resolution transmission electron microscopy (HRTEM) was performed on a JEOL 2010F HRTEM STEM and EELS performed on a Nion UltraSTEM operating at $200 \mathrm{kV}$ with a Gatan Enfinium ER EEL spectrometer. 


\section{Results and Discussion}

In our previous report of the synthesis of planar gold nanotriangles and nanohexagons (Au NT and NH) decorated with SPIONs, TEM analysis showed that $75 \%$ of the observed gold morphologies consisted of NT $(60 \%)$ and $\mathrm{NH}(15 \%)$, and the remaining nanoparticles were a mixture of platonic structures (23\%) and smaller rounded particles (2\%)31. The report also demonstrated that this synthetic approach offers a straightforward and fast route to produce gold planar heterostructures with a $10 \mathrm{wt} \%$ magnetic fraction and with the LSPR band in the NIR region31. These heterostructures are formed by maghemite nanoparticles ( $(\mathrm{W}$ - $\mathrm{Fe} 2 \mathrm{O} 3)$ superparamagnetic at room temperature that can self-assemble as a monolayer in liquid-air interfaces. Figure 1 contains a schematic representation of the synthesis and the physical and chemical characterization of the as-obtained nanostructures.

Bright field (BF) STEM images of a Au NT-SPIONs and a Au NH- SPIONs are shown in Figure 2. It is seen that the Au NTs are of well-defined equilateral shape with their surface decorated by a monolayer of SPIONs as shown in Figures $2 \mathrm{a}$ and $2 \mathrm{~b}$. The equivalent images of a SPIONs decorated $\mathrm{Au} \mathrm{NH}$ are shown in Figures $2 \mathrm{c}$ and $2 \mathrm{~d}$. The resolution of the BF images Figs $2 \mathrm{~b}$ and $2 \mathrm{~d}$ are high enough to see the lattice planes of the SPIONs, and the insets contain the fast Fourier transform (FFT) of their respective images. In the FFTs, each spot indicates a different crystal plane, and a circular ring pattern is an indication of many non-aligned crystal grains/nanoparticles. The circular FFT pattern is observed in both the NT and NH, indicating that the SPIONs are randomly oriented with respect to the Au NT, and no epitaxial relationship between the two exist.

To investigate the composition of the nanotriangles, EEL spectrum imaging (SI) was performed and then analysed using multiple-linear least squares (MLLS) fitting. Figure 3 displays the composition map of a Au NT by MLLS-EELS mapping. Figure 3a shows a high-angle annular dark field (HAADF) image of the nanoparticle, and $3 b$ shows the elemental composition, where red is iron, blue is gold, and yellow is carbon, 3c-3e show each of the individual compositional maps respectively. Figure $3 f$ contains the reference spectra used for MLLS fitting. The fit region is from $45 \mathrm{eV}$ to $65 \mathrm{eV}$ due to similar positions of the bulk plasmons for iron and carbon in the 15 to $40 \mathrm{eV}$ band. Due to the presence of the prominent Fe- $M$ edge at $54 \mathrm{eV}$ and the significantly different low-loss character of gold, the $45-65 \mathrm{eV}$ range presents three completely different spectra that are suitable for an MLLS fit. 
It is important to note that both the gold and iron map show high intensities along the edges and weak/no intensity in the bulk. This is due to the thickness of the gold in the bulk of the nanoparticle, which scatters a large portion of the beam to away from the EELS detector. As a result, in thick samples of strongly scattering materials such as gold, the EELS intensity is reduced. Even in planar structures, such as the NHs and NTs have a three-dimensional character, which can become quite thick. EELS log-ratio thickness measurements show that the NT are $138 \mathrm{~nm}$ thick. Such thicknesses are sufficient to result in reduced signal in the nanoparticle across the entire EEL spectrum.

Due to the fact that faceted edges of the NT taper to a point at the edges, the elemental composition of the Au-NT SPIONs can be observed and analysed at thinner regions.thickness. Figure $3 g$ shows a magnified view of the edge of the structure outlined in Figure 3a. Three regions are indicated: the SPION, the faceted edge, and the bulk of the NT. Figure $3 \mathrm{~h}$ shows low loss EEL spectra from each of those three regions. Points 1 and 3 each show the characteristic low-loss spectra of iron-oxide and gold respectively; however, at Point 2 we see a low-loss spectrum that appears to be a linear combination of the iron and gold spectra. This indicates that even where the iron EELS signal cannot be detected due to scattering from the thick gold, there are SPIONs present on all facets of the NT.

To assess crystallinity in thin structures, the STEM can be used with a defocused probe to generate a 'Ronchigram' to assess crystallinity. In the Ronchigram, diffraction in the sample results in a Kikuchi pattern ${ }^{34}$. Figure 4 a shows a HAADF image of an Au NH-SPIONs nanostructure. The sample is tilted so that the (111) zone axis is parallel to the beam, and the Kikuchi pattern for the (111) zone axis is shown uninterrupted across the entire NH in Figure $4 \mathrm{~b}$, indicating that the entire structure is a single crystal. For the thicker structures where the Kikuchi pattern is not easily observed, crystallinity can be determined through convergent beam electron diffraction (CBED). Figure 4c shows a HAADF image of a thicker structure where no strong Kikuchi pattern is observed. Figure $4 \mathrm{~d}$ and $4 \mathrm{e}$ show CBED patterns from the gold bulk and the thin carbon supporting film. A $16 \times 16$ region including the NT is scanned, and the HAADF intensity along with CBED patterns are collected at each position. Figure $4 \mathrm{f}-4 \mathrm{~h}$ show the resulting maps, where $4 \mathrm{f}$ shows the HAADF map, $4 \mathrm{~g}$ shows the map from the amorphous carbon diffraction ring seen in $4 \mathrm{e}$ (green squares), and $4 \mathrm{~h}$ shows the most intense diffraction spots from the gold (111) pattern (red squares). From the maps we can see that the same (111) CBED pattern is present at all points in the structure and no shift in the diffraction pattern is observed, indicating that the entire NT is a single crystal.

SPIONs were easily dissolved from the Au NPs surface using a diluted $\mathrm{HCl}$ aqueous solution 
( $1: 10$ dilution, $\mathrm{pH} \approx 3)$. Figure 5 shows Au NT-SPIONs before and after the treatment. 5 a shows a NT with the SPIONs still adhered to the surface, while $5 b$ shows a NT without any SPIONs. The SPIONs are visible in the HAADF image presented in $5 a$, but none are observed in $5 b$. To further demonstrate the absence of iron from the treated NTs, the EELS core-loss data from the Fe-L3 edge was collected from a large region at the bottom edge of both NTs. In the NT- SPIONs the Fe-L3 Edge is easily observed, but is completely absent from the NT post-treatment with $\mathrm{HCl}$, indicating that SPIONs have been removed. The ability to dissolve the SPIONs post synthesis opens the door to investigate to which extent the plasmonic modes of single platelet are affected by the dielectric media modification induced by the SPIONs.

Next, insight on the gold nanocrystal nucleation and growth mechanisms is provided. In the polyol synthesis, the heating of $\mathrm{HAuCl}_{4}$ in EG in the presence of PVP leads to the reduction of $\mathrm{Au}(\mathrm{III})$ to $\mathrm{Au}(0)$, where EG serves as solvent and reducing agent and PVP as stabilizer. In our route, PVP-SPIONs are also placed in the solution. They are negatively charged (Zpotential = $30 \mathrm{mV}$ ) exposing the $\mathrm{OH}^{-}$group of the PVP. Under the effect of microwave radiation, the polar $\mathrm{OH}^{-}$rich zones of the SPIONs surface likely serve as hot spots and thus preferential sites where the gold nucleate, similarly as we reported for the in- situ synthesis of other inorganic nanoparticles on hydroxyl rich substrates ${ }^{35,36}$. As a result, gold seeds nucleate in the presence of SPIONs and are attached to them via PVP. Those initial gold seeds will grow from the later reduced gold atoms, resulting in gold particles decorated by a thin layer of SPIONs. However, an explanation why triangular nanocrystals are the most common geometry is still missing. The shape outcome is determined by thermodynamic parameters (e.g., reduction potential and surface capping) and kinetic parameters (e.g., concentration, mass transport, temperature, and the involvement of foreign species) which are intimately and intricately entangled to each other 37,38 . As pointed out recently in a review by Xia et al.39, it is extremely difficult to track alterations to the nuclei at initial crystallization stages due to the lack of experimental tools capable of identifying and monitoring these structures during growth. Nevertheless, the prevalence of planar structures in our case is probably a consequence of a slow initial reduction rate40, may be due to the rather low set-up temperature of 120 o

C. However, this argument is not entirely convincing since higher local temperatures may be present in the MW route. Another possibility is that, as an oxidative etchant, the $\mathrm{Fe}(\mathrm{II}) / \mathrm{Fe}(\mathrm{III})$ pair is kinetically controlling the morphology of metal nanocrystals by retarding the rate of atom supply from the gold precursor41. 
In order to gain further insights on the SPIONs role on the growth kinetics, we started by assessing the SPIONs coverage on the different facets of the gold structures semiquantitatively. It is expected that during crystal growth, free PVP and PVP-SPIONs will selectively adsorb on the (111) facets rather than the (100) facets ${ }^{39}$. In the $\mathrm{NH}$, the crystal facets enclosing the hexagon are the (111) and (100) families in an alternating sequence. Figure 6 depicts how the preferential bonding of PVP-SPIONs to the (111) facets is empirically determined. The system is imaged in two tilt orientations, termed max and min tilt. In each orientation the sample is tilted to one extreme, which causes one specific facet of the NH to be parallel to the beam, allowing that facet to be imaged effectively. Since the facets are alternating, if a (100) facet is aligned with the beam at max tilt (as seen in Figure 6a), then a (111) facet is aligned with the beam at min tilt (Figure 6b). The tilt is performed such that two congruent facets of the nanoparticle are able to be seen at both max and min tilt, and due to the alternating pattern of the (100) and (111) facets, this results in the imaging of two different (100) facets (max- right, min-left) and two different (111) facets (max-left, min- right). HAADF images for a $\mathrm{NH}$ at max and min tilt are shown in Figures $6 \mathrm{c}$ and $6 \mathrm{~d}$ respectively, and indeed it can be seen that at max tilt, the left side has a high coverage while the right side is sparse, and at min tilt the inverse is true. To help visualize the coverage, line profiles from the max and min tilt (shown on Figure 6e and $6 f$ respectively) are performed, and the coverage analysed.

The coverage is calculated the percent of the line profile with HAADF intensity above a threshold, $20 \%$ of the maximum HAADF intensity is chosen qualitatively as giving the best representation of the nanoparticle coverage. In Figures 6g-6j the four line profiles are plotted, showing high coverage at max-left and min-right, and low coverages at max-right and min-left. It is worth noting that in the min-left profile (Fig 6i) the coverage is artificially increased due to a large portion of the supporting carbon film that overlaps with the NH edge. Determining which crystal axis corresponds to the high- concentration facets is not possible from the STEM data, as the tilt limits of the STEM holders are $\sim 20^{\circ}$, which is only enough to preferentially align a facet, but not to tilt into a crystal zone access normal to the facet. As a result, the data is not sufficient to absolutely confirm that SPIONs selectively bond to the $<111\rangle$ it does provide direct experimental evidence of preferential bonding on different gold facets in the Au-NH SPIONs ${ }^{39}$.

Finally, in Figure 7, EELS fine structure along with quantitative analysis of the relative composition of iron and oxygen are used to determine the phase of the iron oxide present in the SPIONs. Figure $7 \mathrm{a}$ and $7 \mathrm{~b}$ show the $\mathrm{O}-K$ and Fe- $L$ edges taken from three different samples: a reference sample of $\mathrm{Fe}_{2} \mathrm{O} 3$ (with $3+$ valence), a reference sample of $\mathrm{FeO}$ (with 2+ valence), 
and finally the SPIONs. In Fig. 7a the O-K edge from the three samples are shown. The pre-peak at $529 \mathrm{eV}$ shows a clear distinction between $\mathrm{Fe} 2 \mathrm{O} 3$ and $\mathrm{FeO}$, and more importantly, that the pre-peak in the SPIONs is almost an exact match of the Fe2O3. Additionally, in the Fe- $L$ edge EELS shown in Fig. 7b, it can be seen that the peak energy of $\mathrm{Fe} 2 \mathrm{O} 3$ and $\mathrm{FeO}$ differ significantly $(2+$ at $706 \mathrm{eV}, 3+$ at $708 \mathrm{eV})$, and that the SPIONs peak clearly aligns with the Fe2O3 peak at $708 \mathrm{eV}$. Moreover, it is also observed that the $\mathrm{FeO} L 2$ edge has only a single peak at $718 \mathrm{eV}$ while both the SPIONs and the $\mathrm{Fe} 2 \mathrm{O} 3$ have two peaks at $719 \mathrm{eV}$ and $721 \mathrm{eV}$. There are some dissimilarities between the $\mathrm{Fe}_{2} \mathrm{O} 3$ reference sample and the SPIONs, namely a stronger prepeak on the $L 3$ edge and a larger area beneath the $L 2$ edge. The likely reason for these differences is sample damage from the electron beam.

However, other iron-oxide phases besides $\mathrm{Fe}_{2} \mathrm{O} 3$ and $\mathrm{FeO}$ exist and in order to further investigate the SPIONs composition, we quantify the relative amounts of iron and oxygen in the SPIONs. The relative concentration is determined by fitting and then subtracting the power-law EELS background and then integrating the intensity of the tails of the oxygen and iron EELS edges $^{42}$. The near-edge fine structure varies significantly across different compounds, so these spectral regions are excluded from the integration. X-ray photo-absorption (XRPA) cross sections are known to be excellent analogues of the corresponding EELS cross sections, and the intensity of the EELS signal is determined by fitting these XRPA cross sections to the power-law tails of the EELS edges ${ }^{43}$. A quantitative model of the EELS signal is formed and plotted against the experimental EELS signal in Fig. 7c, where it can be seen that the quantitative model matches well the power-law tails of the edges and ignores the variations due to fine-structure. From the quantitative model a relative composition of $39.25 \% \mathrm{Fe}$ and $60.75 \% \mathrm{O}$ is determined, which corresponds to the composition of $\mathrm{Fe}_{2} \mathrm{O} 3$ and is in agreement with the fine- structure analysis in Fig. 7a and 7b. These results indicate that the Fe retains its Fe(III) character and does not reduce to Fe(II). 


\section{Conclusions}

Here, we presented nanoscale structural and compositional characterization of gold planar nanostructures (triangles and hexagons) decorated with superparamagnetic maghemite nanoparticles. The magnetic gold planar structures are synthesized through 10 minutes of microwave heating, are dispersible in water, display the LSPR band in the NIR region, and can have the magnetic nanoparticles easily removed from the gold surface using a diluted acidic solution. High spatial- resolution STEM analysis determines that the gold structures are all single crystals and that the SPIONs adhere across the entire gold nanostructures with no epitaxial relationship. However, the different gold facets are shown to interact differently with the PVP polymer, changing the coverage of the SPIONs on the different facets. As a result of the differences in facet coverage, the SPIONs could be further exploited as a probe to follow initial seed formations through the nanocrystal growth process, which are difficult to observe directly. Finally, the phase of the iron oxide in the SPIONs was determined through STEM-EELS to be $\mathrm{Fe} 2 \mathrm{O} 3$, ruling out that the SPIONs undergo a reduction from $\mathrm{Fe}(\mathrm{III})$ to $\mathrm{Fe}$ (II) during the synthesis of the gold planar structures.

\section{Acknowledgements}

This research was partially funded by the Spanish Ministry of Economy and Competitiveness in co-funding with European Social Funds through the MAT2012-35324 project, the Severo Ochoa Programme for Centers of Excellence in R\&D (SEV-2015- 0496) and the Ramón y Cajal program (AL, RyC-2010-06082) and the Generalitat de Catalunya (2014SGR213). Authors also acknowledge support of the Chinese Scholarship Council (SMY, 201206150053) and the following projects: COST Action MP1202, and the U.S. Department of Energy grant DE-FG0209ER46554 (JAH, STP). Work at Oak Ridge National Laboratory was supported by the Department of Energy, Office of Science, Basic Energy Sciences, Materials Sciences and Engineering Division. 


\section{References}

1 E. Taboada, R. Solanas, E. Rodríguez, R. Weissleder, A. Roig, Advanced Functional Materials, 2009, 19, 2319.

2 S. Guo, D. Li, L. Zhang, J. Li, E. Wang, Biomaterials, 2009, 30, 1881.

3 E. Carenza, V. Barceló, A. Morancho, J. Montaner, A. Rosell, A. Roig, Acta Biomaterialia, 2014, 10, 3775.

4 C.-J. Yu, C.-Y. Lin, C.-H. Liu, T.-L. Cheng, W.-L. Tseng, Biosensors and Bioelectronics, 2010, 26, 913.

5 R. Di Corato, A. Espinosa, L. Lartigue, M. Tharaud, S. Chat, T. Pellegrino, C. Ménager, F. Gazeau, C. Wilhelm, Biomaterials 2014, 35, 6400.

6 G. Luke, D. Yeager, S. Emelianov, Annals of Biomedical Engineering, 2012, 40, 422.

7 L. Xu, H. Kuang, C. Xu, W. Ma, L. Wang, N.A. Kotov, Journal of the American Chemical Society, 2011, 134, 1699.

8 B. Duncan, C. Kim, V.M. Rotello, Journal of Controlled Release, 2010, 148, 122.

9 L.R. Hirsch, R.J. Stafford, J.A. Bankson, S.R. Sershen, B. Rivera, R.E. Price, J.D. Hazle, N.J. Halas, J.L. West, Proceedings of the National Academy of Sciences, 2003, 100, 13549.

10 N.G. Bastús, J. Comenge, V.F. Puntes, Langmuir, 2011, 27, 11098.

11 J.S. DuChene, W. Niu, J.M. Abendroth, Q. Sun, W. Zhao, F. Huo, W.D. Wei, Chemistry of Materials, 2012, 25, 1392.

12 M. Smith, M. C. Mancini, S. Nie, Nature Nanotechnology 2009, 4, 710.

13 L. Scarabelli, M. Coronado-Puchau, J.J. Giner-Casares, J. Langer, L.M. Liz-Marzán, ACS Nano, 2014, 8, 5833.

14 B. Pelaz, V. Grazu, A. Ibarra, C. Magen, P. del Pino, P.; J.M. de la Fuente, Langmuir, $2012,28,8965$.

15 M. Perez-Hernandez, P. del Pino, S.G. Mitchell, M. Moros, G. Stepien, B. Pelaz, W.J. Parak, E.M. Galvez, J. Pardo, J.M. de La Fuente, ACS Nano 2015, 27 9(1), 52.

16 T.K. Sau, C.J. Murphy, Journal of the American Chemical Society, 2004, 126, 8648.

17 J.E. Millstone, G.S. Métraux, C.A. Mirkin, Advanced Functional Materials, 2006, 16, 1209.

18 B. Wiley, Y. Sun, B. Mayers, Y. Xia, Chemistry - A European Journal, 2005, 11, 454. 
19 I. Washio, Y. Xiong, Y. Yin, Y. Xia, Advanced Materials, 2006,

18, 1745 .

30 Y. Sun, Y. Xia, Science, 2002, 298, 2176.

31 S.-M.Yu, J. A. Hachtel, M. F. Chisholm, S. T. Pantelides, A. Laromaine, A. Roig, Nanoscale, 2015, 7, 14039.

32 O. Pascu, E. Carenza, M. Gich, S. Estradé, F. Peiró, F.; G. Herranz, A. Roig, The Journal of Physical Chemistry C, 2012, 116, 15108.

33 S.-M Yu, A. Laromaine, A. Roig, J. Nanopart. Res., 2014, 16, 1. 34 D.B. Williams, C.B.

Carter, Transmission Electron Microscopy, Springer, USA, 1996.

35 O. Pascu, J.M. Caicedo, M. Lopez-Garcia, V. Canalejas, A. Blanco, C. Lopez, J. Arbiol, J. Fontcuberta, A. Roig, G. Herranz, Nanoscale, 2011, 3, 4811.

36 M. Zeng, A. Laromaine, W. Feng, P.A. Levkin, A. Roig, Journal of Materials Chemistry C, 2014, 2, 6312 .

37 N. Malikova, I. Pastoriza-Santos, M. Schierhorn, N.A. Kotov, L.M. Liz-Marzán, Langmuir, 2002, 18, 3694.

38 Y. Xiong, I. Washio, J. Chen, M. Sadilek, Y. Xia, Angewandte Chemie International Edition, 2007, 46, 4917.

39 Y. Xia, X. Xia, H.-C. Peng, J. Liu, Journal of the American Chemical Society, 2015, 137, 7947.

40 Y. Wang, H.-C. Peng, J. Liu, C. Z. Huang, and Y. Xia, Nano Letters, 2015, 15, $1445-$ 1450.

41 J. Chen, T. Herricks, Y. Xia, Angewandte Chemie International Edition, 2005, 44, 2589.

42 R.F. Egerton., Rep. Prog. Phys., 2009, 72, 016502.

43 R.F. Egerton, Ultramicrsocopy, 1993, 50, 13-28. 

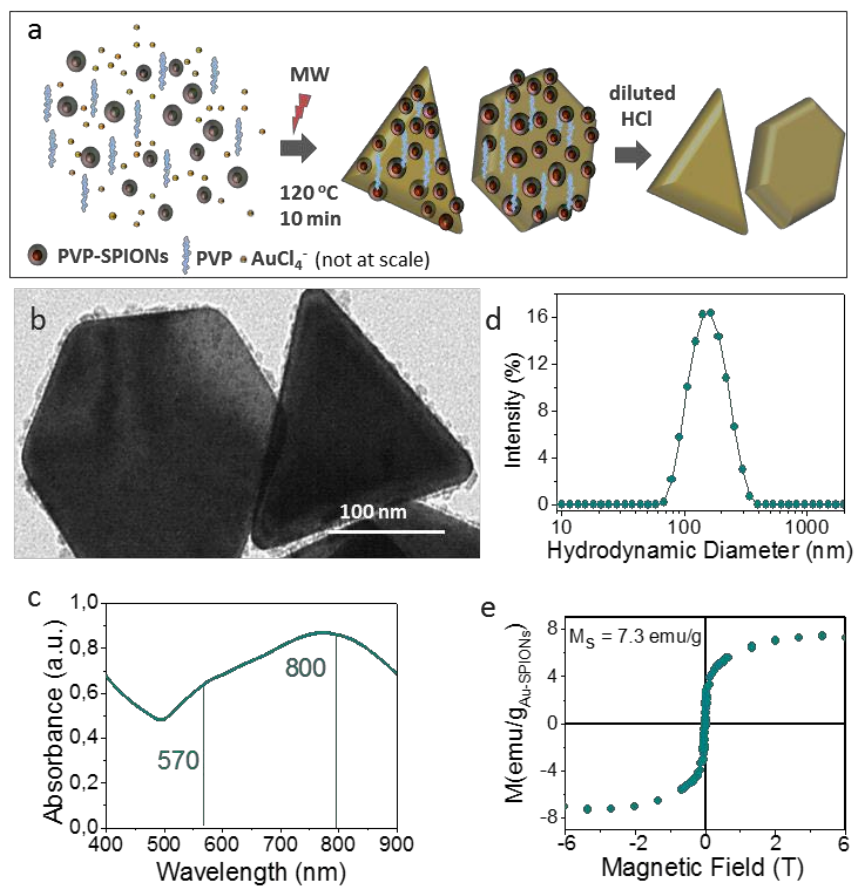

Figure 1. (a) Schematic representation of the synthesis route.

(b) HRTEM image of a Au NH-SPIONs and a Au NT-SPIONs. Characterization of the heterostructures: (c) UV-Vis-NIR spectra. (d) Hydrodynamic diameter of the Au-SPIONS measured by DLS. (e) Magnetization curve up to $6 \mathrm{~T}$ at $5 \mathrm{~K}$.
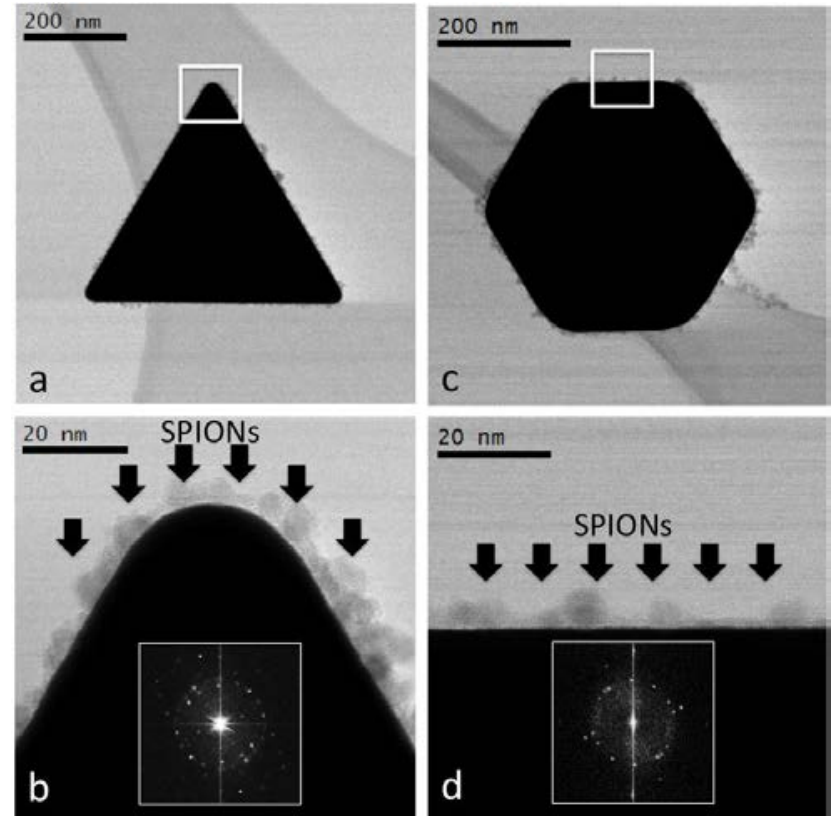

Figure 2. (a) Bright-field (BF) scanning transmission electron microscopy (STEM) image of a single Au NT-SPIONs nanostructure. (b) BF-STEM image of NT tip outlined in (a) showing crystallinity of the SPIONs. The inset contains an fast Fourier transform (FFT) of the BF image and shows FFT spots arranged in a circular pattern indicating that the SPIONs are randomly oriented with respect to the NT.(c) and (d) BF-STEM images of a Au NH-SPIONs structure and the top edge containing the SPIONs. In the inset of (d) the same circular FFT pattern is visible, indicating no epitaxial relationship between the Au and SPIONs occur in either geometry. 

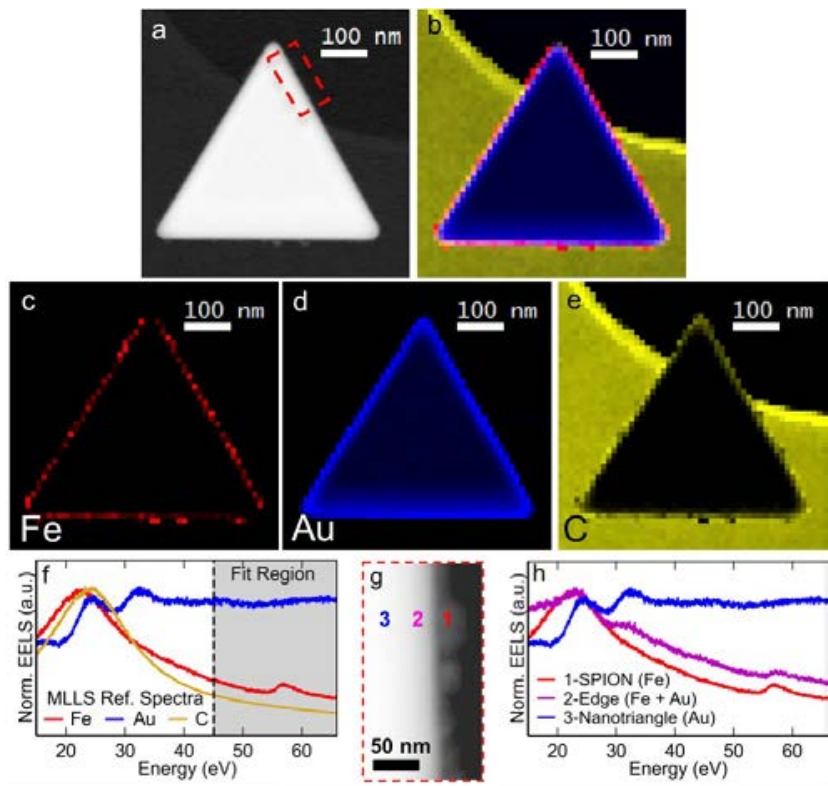

Figure 3. (a) High angle annular dark field (HAADF) image of an Au NT-SPIONs composite. (b) The elemental map of the Au NT- SPIONs composed of the EEL spectrum image (SI) maps of Fe, Au, and C (shown in (c)-(e) respectively) determined through multiple linear least squares (MLLS) fitting. (f) The MLLS reference spectra used for the fitting. Fit region is $45 \mathrm{eV}$ through $65 \mathrm{eV}$ to avoid the similar bulk plasmons of Fe and $\mathrm{C}$.

(g) Close up of the outlined region in (a), showing the three regions: the Fe in SPION, the faceted edge of the structure, and the thick bulk of the NT. (h) Spectra from each of the three regions highlighted in (g), showing that the signal from the faceted edge is a combination of $\mathrm{Fe}$ and $\mathrm{Au}$ and that the coverage of the nanoparticles is uniform across all the facets.
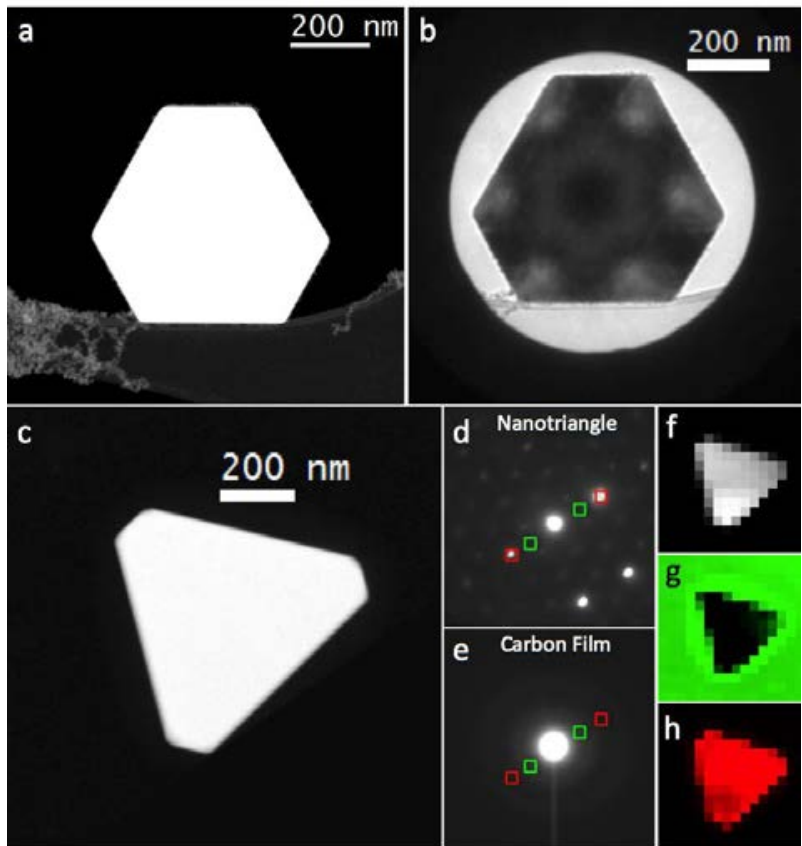

Figure 4. (a) HAADF image of a Au NH-SPIONs structure. (b) STEM Ronchigram of the NH aligned such with the (111) crystal axis parallel to the beam. The (111) Kikuchi diffraction pattern can be observed uninterrupted across the entire structure, indicating that the NH is a single crystal. (c) HAADF image of an Au NT, too thick for observing the Kikuchi pattern. (d) and (e) convergent beam electron diffraction (CBED) pattern from the Au NT (d) and the support C film (e) for the Au NT of (c). (f)-(h) 16×16 maps of the nanotriangle are made using the HAADF intensity (f), the intensity at the amorphous $C$ diffraction ring

(g) (green squares in (d) and (e)), and (h) the Au (111) diffraction spots (red squares). The entire NT shows the same (111) diffraction pattern, indicating that is a single crystal. 

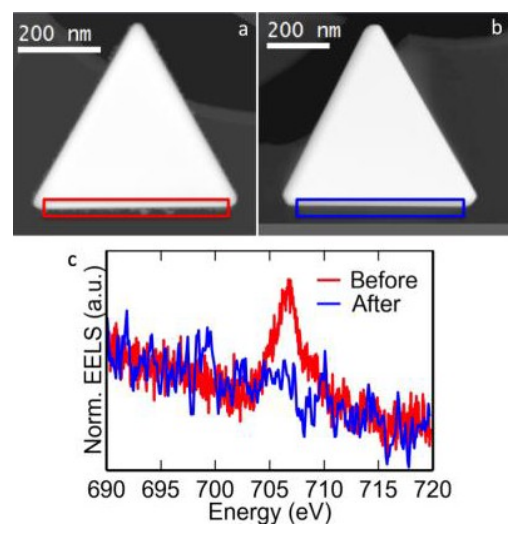

Figure 5. HAADF images of different Au NT-SPIONs from before (a) and after (b) the SPION removal treatment. EEL spectra are collected and summed over the outlined region in both images and plotted in (c). In the 'before' EELs the

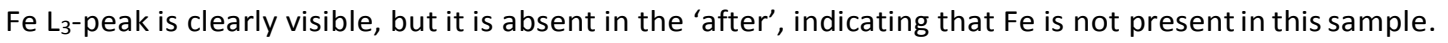
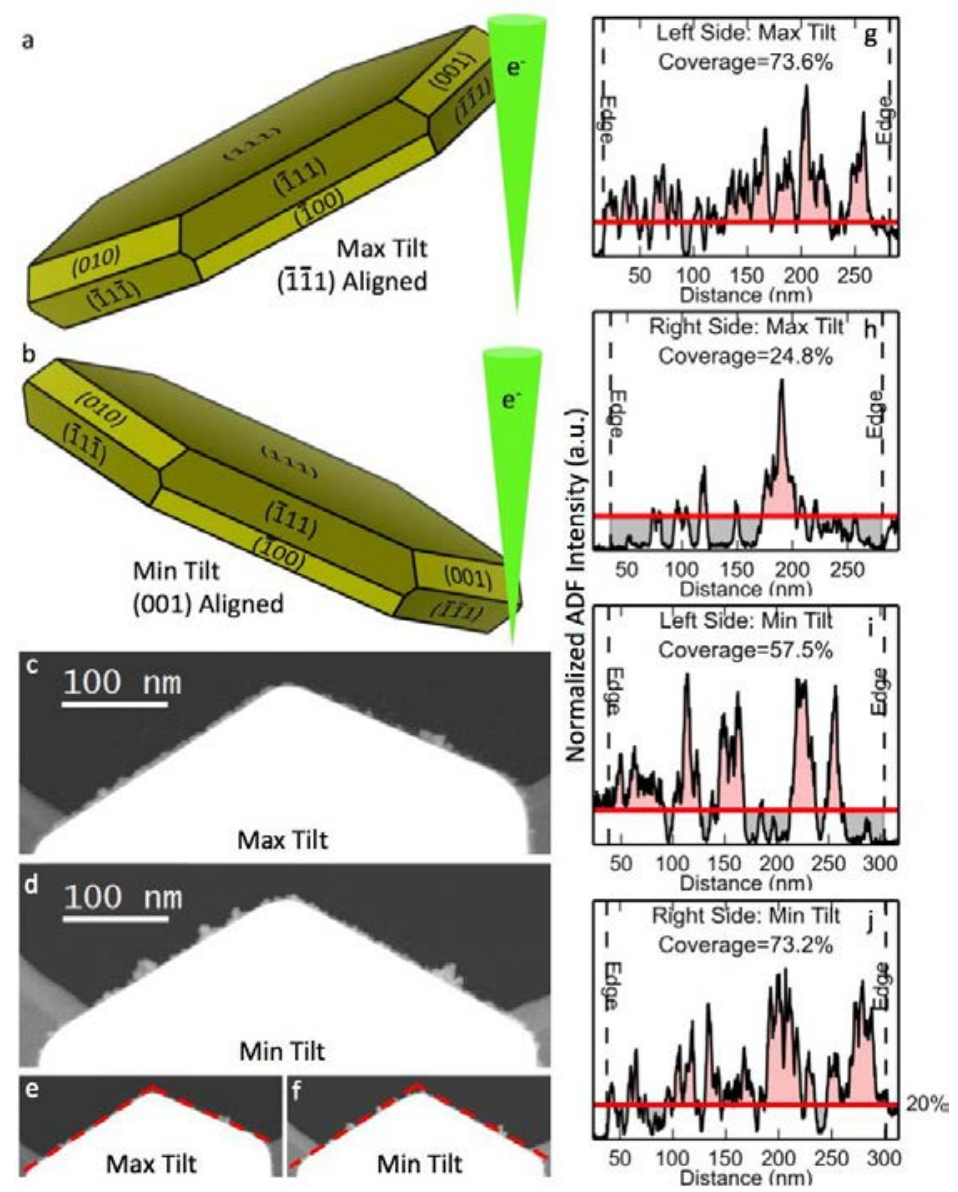

Figure 6. Preferential bonding of the SPIONs to a specific facet is determined through tilting the sample. (a) and (b) show two orientations of the $\mathrm{NH}$ with respect to the beam, termed max and min tilt respectively, which align 
alternating facets with the electron beam. HAADF images of the max (c) and $\min (d)$ tilts show that on alternating facets there are varying concentrations of the SPIONs. Line profiles along the edges of the two tilts, (e)-max and (f)-min) are plotted and the percent coverage is calculated by seeing where along the line profile the HAADF intensity is greater than a $20 \%$ threshold (red-line). (g-j) Show the line profiles on the two different edges of the $\mathrm{NH}$ at the two tilts, and it can be seen that at max-left $(\mathrm{g})$ and min-right (j) the coverage is significantly higher than max-right

(h) and min-left (i). Due to the alternating nature of the facets, this demonstrates that SPIONs preferentially bond to one facet of the $\mathrm{NH}$.
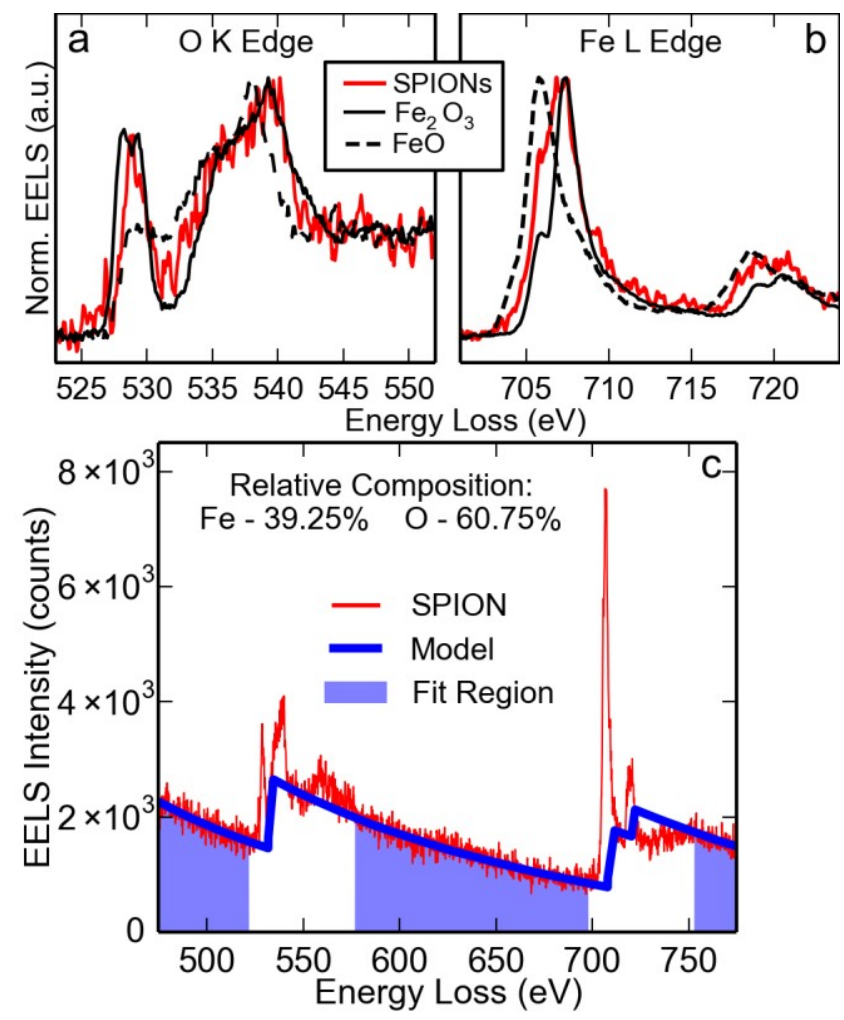

Figure 7. (a) O- $K$ edge EELS. The two reference samples $\left(\mathrm{Fe}_{2} \mathrm{O}_{3}\right.$ - solid, $\mathrm{FeO}$-dashed) show two distinct behaviours in the relative intensity of the pre-peak at $529 \mathrm{eV}$, the SPIONs (red) match the $\mathrm{Fe}_{2} \mathrm{O}_{3}$. (b) Fe- $L$ edge EELS. A $2 \mathrm{eV}$ shift (708 eV- $\mathrm{Fe}_{2} \mathrm{O}_{3}, 706 \mathrm{eV}-\mathrm{FeO}$ ) is observed in the peak intensity of the $L$ edge, and the SPIONs share the $\mathrm{Fe}_{2} \mathrm{O}_{3}$ at $708 \mathrm{eV}$. (c) Relative composition of $\mathrm{O}$ and Fe. By fitting $\mathrm{x}$-ray photo-absorption cross sections to the tails of the $\mathrm{O}$ and Fe edges, the intensities of the two edges can be directly compared and the relative composition calculated. The result is a 1.548 ratio between $\mathrm{O}$ and $\mathrm{Fe}$, further confirming the SPIONs are in the $\mathrm{Fe}_{2} \mathrm{O}_{3}$ phase. 
